$03,11,06$

\title{
Дипольное упорядочение и ионная проводимость в NASICON-подобных структурах типа $\mathrm{Na}_{3} \mathrm{Sc}_{2}\left(\mathrm{PO}_{4}\right)_{3}$
}

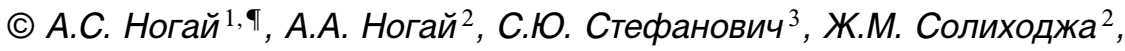 \\ Д.Е. Ускенбаев 1 \\ ${ }^{1}$ Казахский агротехнический университет им. С. Сейфуллина, \\ Астана, Казахстан \\ ${ }^{2}$ Евразийский Национальный университет им. Л.Н. Гумилева, \\ Астана, Казахстан \\ ${ }^{3}$ Научно-исследовательский физико-химический институт им. Л.Я. Карпова, \\ Москва, Россия \\ IE-mail: nogay06@mail.ru
}

Поступила в Редакцию 27 февраля 2019 г.

В окончательной редакции 4 июня 2019 г.

Принята к публикации 4 июня 2019 г.

Изучены вопросы дипольного упорядочения и ионной проводимости поликристалла $\mathrm{Na}_{3} \mathrm{Sc}_{2}\left(\mathrm{PO}_{4}\right)_{3}$, который имеет три полиморфные фазы: $\alpha, \beta, \gamma$. Уточнены особенности строения кристалла $\alpha-\mathrm{Na}_{3} \mathrm{Sc}_{2}\left(\mathrm{PO}_{4}\right)_{3}$, a также дипольного упорядочения и релаксационной поляризации в $\alpha$ - и $\beta$-фазах. Возникновение дипольного упорядочения в $\alpha$-фазе и частичного разупорядочения в $\beta$-Na3 $\mathrm{Sc}_{2}\left(\mathrm{PO}_{4}\right)_{3}$, а также высокой ионной проводимости в $\beta$ - и $\gamma$-фазах поликристалла $\mathrm{Na}_{3} \mathrm{Sc}_{2}\left(\mathrm{PO}_{4}\right)_{3}$ связано с фазовыми превращениями $\alpha \rightarrow \beta, \beta \rightarrow \gamma$, приводящими к структурным изменениям ромбоэдрического кристаллического каркаса $\left\{\left[\mathrm{Sc}_{2}\left(\mathrm{PO}_{4}\right)_{3}\right]^{3-}\right\}_{3 \infty}$. Предложена модель, поясняющая явления дипольного упорядочения и ионной проводимости в $\mathrm{Na}_{3} \mathrm{Sc}_{2}\left(\mathrm{PO}_{4}\right)$.

Ключевые слова: поликристалл, ионная проводимость, фазовые переходы, кристаллический каркас, диэлектрическая фаза, суперионная фаза.

DOI: 10.21883/FTT.2019.11.48401.395

\section{1. Введение}

Изоструктурным аналогом $\mathrm{Na}_{3} \mathrm{Cr}_{2}\left(\mathrm{PO}_{4}\right)_{3}$ в семействе NASICON является $\mathrm{Na}_{3} \mathrm{Sc}_{2}\left(\mathrm{PO}_{4}\right)_{3}$, который обладает низкой ионной проводимостью в низкотемпературной $\alpha$-фазе и высокой ионной проводимостью в высокотемпературных $\beta$ - и $\gamma$-фазах [1].

Несмотря на то, что кристаллическая структура и свойства двойного фосфата натрия-скандия $\mathrm{Na}_{3} \mathrm{Sc}_{2}\left(\mathrm{PO}_{4}\right)_{3}$ изучалась многократно различными учеными [2-12], до сих пор нет однозначности как в вопросах, связанных с описанием температур фазовых переходов, структурных и проводящих параметров этого соединения, так и в вопросах трактовки дипольного упорядочения. В этой связи дальнейшее изучение особенностей появления диэлектрических свойств и электропроводности $\mathrm{Na}_{3} \mathrm{Sc}_{2}\left(\mathrm{PO}_{4}\right)_{3}$ является вполне актуальным. Кристаллы с ромбоэдрическими каркасами $\left\{\left[\mathrm{M}_{2}\left(\mathrm{PO}_{4}\right)_{3}\right]^{3-}\right\}_{3 \infty}$ семейства NASICON уже широко применяются как конструкционные материалы в источниках тока [13].

Целью настоящей работы является установление взаимосвязи структурных особенностей с появлением дипольного упорядочения, ионного и суперионного состояния в различных полиморфных модификациях $\mathrm{Na}_{3} \mathrm{Sc}_{2}\left(\mathrm{PO}_{4}\right)_{3}$.

\section{2. Методика эксперимента}

Получение поликристаллов $\mathrm{Na}_{3} \mathrm{Sc}_{2}\left(\mathrm{PO}_{4}\right)_{3}$ было осуществлено твердофазным синтезом по керамической технологии из шихты, получаемой при смешивании исходных реактивов: $3 \mathrm{Na}_{2} \mathrm{CO}_{3}+2 \mathrm{Sc}_{2} \mathrm{O}_{3}+6 \mathrm{NH}_{4} \mathrm{H}_{2} \mathrm{PO}_{4}$, взятых в стехиометрических соотношениях, путем двухстадийного отжига. Первый отжиг проводили при $870 \mathrm{~K}$, а второй при $970 \mathrm{~K}$ с дополнительными гомогенизирующими перетираниями. Время отжига образцов на каждой стадии твердофазного синтеза составляло $8 \mathrm{~h}$.

Фазовая принадлежность и структурные параметры поликристаллических образцов $\mathrm{Na}_{3} \mathrm{Sc}_{2}\left(\mathrm{PO}_{4}\right)_{3}$ были исследованы рентгенографическим методом порошка с использованием дифрактометра ДРОН-3 (CuK $K_{\alpha}$-излучение).

Нелинейно-оптические свойства поликристалла $\mathrm{Na}_{3} \mathrm{Sc}_{2}\left(\mathrm{PO}_{4}\right)_{3}$ определялись методом генерации второй оптической гармоники (ГВГ) от неодимового лазерного излучения.

Определение проводящих и диэлектрических свойств проводили на хорошо спеченных образцах (с плотностью 96\% от теоретической) методом импедансной спектроскопии с помощью импедансметра ВМ-507 в интервале температур $293-573 \mathrm{~K}$ и в диапазоне частот $5-5 \cdot 10^{5} \mathrm{~Hz}$. Диэлектрические характеристики образцов 
изучали с помощью прибора РИПСЭ-М на частоте $2 \mathrm{GHz}$. Для создания электрода на образец наносили палладий, который рассматривался как идеально блокирующий электрод.

\section{3. Результаты и обсуждение}

\section{1. Результаты синтеза и рентгеновского исследования поликристалла $\mathrm{Na}_{3} \mathrm{Sc}_{2}\left(\mathrm{PO}_{4}\right)_{3}$}

Синтезированные поликристаллы $\mathrm{Na}_{3} \mathrm{Sc}_{2}\left(\mathrm{PO}_{4}\right)_{3}$ имели белую окраску, представляли собой таблетки диаметром $15 \mathrm{~mm}$ и толщиной $1 \mathrm{~mm}$.

Рентгенографическими измерениями была установлена однофазность приготовленных образцов. По данным работы [2], это соединение в $\alpha$-фазе имеет моноклинную структуру пространственной группы (пр.гр.) $B в$ с параметрами $a=16.10 \AA, b=9.109 \AA$, $c=8.928 \AA, \gamma=127.15^{\circ}$. В настоящей работе было установлено, что элементарная ячейка поликристалла $\alpha$-фазы $\mathrm{Na}_{3} \mathrm{Sc}_{2}\left(\mathrm{PO}_{4}\right)_{3}$ при комнатной температуре имеет моноклинную структуру с параметрами $a=16.090 \AA, b=9.076 \AA, c=8.956 \AA, \gamma=126.95^{\circ}$. Установленные нами структурные параметры для $\alpha$-фазы $\mathrm{Na}_{3} \mathrm{Sc}_{2}\left(\mathrm{PO}_{4}\right)_{3}$ близки к структурным данным, представленным в работе [2].

\section{2. Результаты теста на нецентросимметричность и исследование ионной проводимости поликристалла $\mathrm{Na}_{3} \mathrm{Sc}_{2}\left(\mathrm{PO}_{4}\right)_{3}$}

Проведение теста на нецентросимметричность поликристалла $\alpha-\mathrm{Na}_{3} \mathrm{Sc}_{2}\left(\mathrm{PO}_{4}\right)_{3}$ (с помощью лазерного излучения) при $T=293 \mathrm{~K}$ позволило выявить наличие достаточно заметного сигнала ГВГ, интенсивность которого составила $I_{2 \omega} / I_{2 \omega} \mathrm{SiO}_{2}=15$ (рис. 1), что характерно для полярных фаз сегнетоэлектрического типа. C повышением температуры наблюдалось достаточно резкое уменьшение интенсивности сигнала ГВГ, а при $T=339 \mathrm{~K}$ величина сигнала спадала до нуля, что указывает на фазовый переход из полярной в неполярную (параэлектрическую) фазу $\alpha \rightarrow \beta$.

Ранее авторами работы [6] была установлена полярность фазы $\alpha-\mathrm{Na}_{3} \mathrm{Sc}_{2}\left(\mathrm{PO}_{4}\right)_{3}$ нейтронографическим методом.

С помощью импедансного метода были измерены ионные проводимости кристаллитов (зерен) поликристалла $\mathrm{Na}_{3} \mathrm{Sc}_{2}\left(\mathrm{PO}_{4}\right)_{3}$, поскольку данная методика позволяет отделить ионную проводимость кристаллитов от проводимости межзеренных прослоек поликристаллов.

Результаты измерения температурной зависимости ионной проводимости кристаллитов поликристалла $\mathrm{Na}_{3} \mathrm{Sc}_{2}\left(\mathrm{PO}_{4}\right)_{3}$ позволяют выделить на зависимости $\sigma T(T)$ три линейных участка, соответствующих трем полиморфным модификациям - $\alpha, \beta, \gamma$ (рис. 2 ).

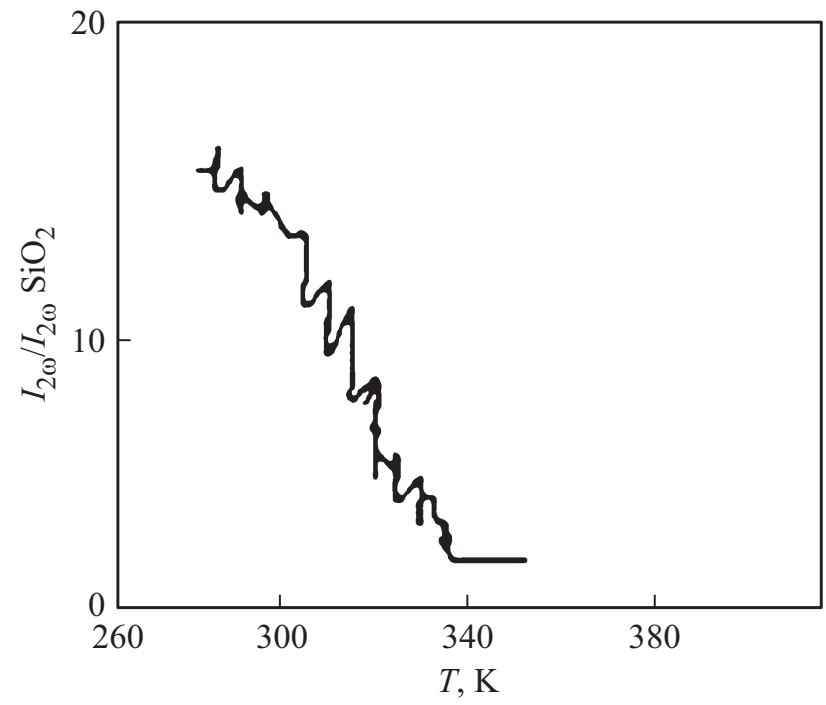

Рис. 1. Температурная зависимость интенсивности генерации второй оптической гармоники лазерного излучения для поликристалла $\mathrm{Na}_{3} \mathrm{Sc}_{2}\left(\mathrm{PO}_{4}\right)_{3}$.

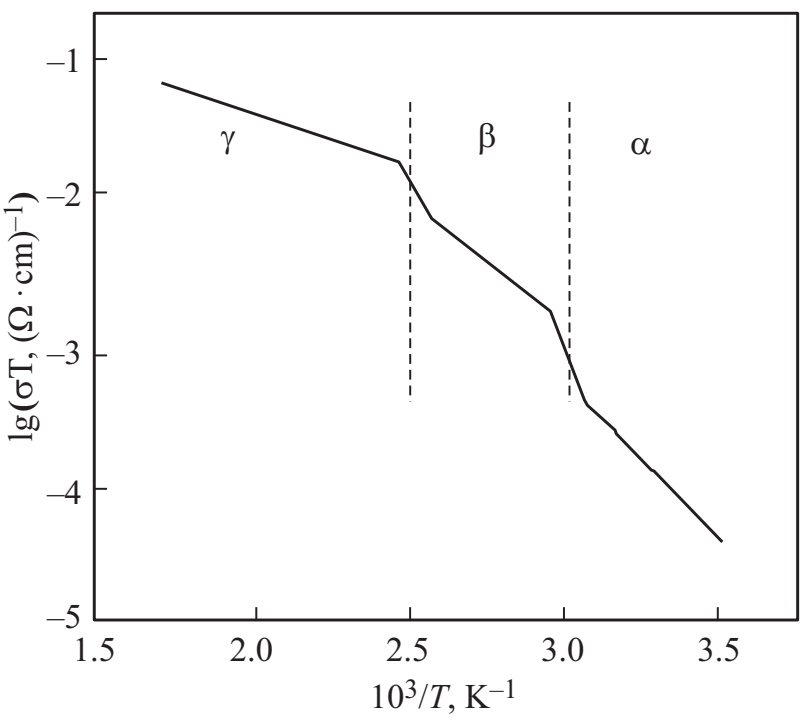

Рис. 2. Температурная зависимость ионной проводимости кристаллитов для поликристаллического образца $\mathrm{Na}_{3} \mathrm{Sc}_{2}\left(\mathrm{PO}_{4}\right)_{3}$. $\mathrm{C}$ помощью штрих-пунктирных линий выделены участки ионной проводимости, относящиеся к $\alpha$-, $\beta$-, $\gamma$-фазам.

Следует отметить, что для поликристаллических образцов характерна изотропность физических свойств, поэтому фазовые переходы на зависимости $\sigma T(T)$ выражены „протяженными“ температурными интервалами, которые соединены линиями в виде „наклонных ступенек“. Однако в случае монокристаллов $\mathrm{Na}_{3} \mathrm{Cr}_{2}\left(\mathrm{PO}_{4}\right)_{3}$ были установлены „прямоугольные ступеньки“ [1], характеризующие резкие скачки проводимости при фазовых переходах. Середина первого „протяженного“ температурного интервала на графике температурной зависимости $\sigma T(T)$ соответствует $T=339 \mathrm{~K}$ (эта температура сов- 
Таблица 1. Параметры ионопереноса для соединения $\mathrm{Na}_{3} \mathrm{Sc}_{2}\left(\mathrm{PO}_{4}\right)_{3}$

\begin{tabular}{c|c|c|c|c}
\hline Фазы & $\begin{array}{c}\text { Ионная проводимость } \sigma, \\
(\Omega \cdot \mathrm{cm})^{-1}\end{array}$ & $\begin{array}{c}\text { Энергия } \\
\text { активации } \Delta E, \mathrm{eV}\end{array}$ & $\begin{array}{c}\text { Типы } \\
\text { фазовых переходов }\end{array}$ & $\begin{array}{c}\text { Температуры } \\
\text { фазовых переходов, } \mathrm{K}\end{array}$ \\
\hline$\alpha$ & $2 \cdot 10^{-5}(293 \mathrm{~K})$ & 0.52 & $\alpha \rightarrow \beta$ & $T_{\alpha \rightarrow \beta}=339$ \\
\hline$\beta$ & $2.4 \cdot 10^{-3}(373 \mathrm{~K})$ & 0.36 & $\beta \rightarrow \gamma$ & $T_{\beta \rightarrow \gamma}=439$ \\
\hline$\gamma$ & $2.5 \cdot 10^{-2}(573 \mathrm{~K})$ & 0.22 & &
\end{tabular}

падает с температурой фазового перехода $T_{\alpha \rightarrow \beta}$ установленным методом ГВГ), что указывает на наличие температурного фазового перехода $\left(T_{\alpha \rightarrow \beta}=339 \mathrm{~K}\right)$ из $\alpha$-фазы в $\beta-\mathrm{Na}_{3} \mathrm{Sc}_{2}\left(\mathrm{PO}_{4}\right)_{3}$. Для фазы $\alpha-\mathrm{Na}_{3} \mathrm{Sc}_{2}\left(\mathrm{PO}_{4}\right)_{3}$ значение ионной проводимости составляет $2 \cdot 10^{-5}(\Omega \cdot \mathrm{cm})^{-1}$ при $293 \mathrm{~K}$, а энергия активации достигает $0.52 \mathrm{eV}$.

Сушественное повышение проводимости $2.4 \cdot 10^{-3}(\Omega \cdot \mathrm{cm})^{-1}$ при $T=373 \mathrm{~K}$ и снижение энергии активации до $0.36 \mathrm{eV}$ при фазовом переходе $\alpha \rightarrow \beta$ можно связать со структурными изменениями кристаллического каркаса $\beta$ - $\mathrm{Na}_{3} \mathrm{Sc}_{2}\left(\mathrm{PO}_{4}\right)_{3}$, сопровождающими этот переход [2-7,14]. Вероятно, фазовый переход $T_{\alpha \rightarrow \beta}$ способствуют частичному снятию моноклинного искажения кристаллического каркаса и увеличению концентрации подвижных ионов натрия в кристалле, способных участвовать в проводимости.

Несмотря на то, что энергия активации достаточно высока для $\beta-\mathrm{Na}_{3} \mathrm{Sc}_{2}\left(\mathrm{PO}_{4}\right)_{3}$, все же заметный скачок проводимости (проводимость увеличивается более чем на порядок) и снижение энергии активации с 0.52 до $0.36 \mathrm{eV}$ при фазовом переходе $\alpha \rightarrow \beta$ позволяет говорить о псевдосуперионном характере проводимости.

Дальнейшее повышение температуры приводит к очередному фазовому превращению при $T_{\beta \rightarrow \gamma}=439 \mathrm{~K}$ (температура перехода была определена так же, как и в первом случае), и соответственно к еще большему увеличению проводимости поликристалла (до $2.5 \cdot 10^{-2}(\Omega \cdot \mathrm{cm})^{-1}$ при $573 \mathrm{~K}$ ), а также снижению энергии активации до $0.22 \mathrm{eV}$, что может быть связано с полным снятием моноклинных искажений кристаллического каркаса в результате фазового перехода $\beta \rightarrow \gamma$. Проводимость поликристалла $\gamma-\mathrm{Na}_{3} \mathrm{Sc}_{2}\left(\mathrm{PO}_{4}\right)_{3}$ уже можно рассматривать как чисто суперионную.

Результаты температурной зависимости проводимости поликристалла $\mathrm{Na}_{3} \mathrm{Sc}_{2}\left(\mathrm{PO}_{4}\right)_{3}$ были установлены на основе достаточно точных воспроизводимых результатов.

Представленную на рис. 2 температурную зависимость электропроводности можно описать следующим соотношением [1]:

$$
\sigma T=A_{i} \sum_{i=1}^{n} \exp \left(-\frac{\Delta E_{i}}{k T}\right),
$$

где $\Delta E_{i}$ - энергия активации проводимости $i$-фаз; $k-$ постоянная Больцмана; $T$ - абсолютная температура; $A_{i}$ - постоянные коэффициенты, характеризующие $i$-фазовые состояния, $n=3$ (число фаз).
Путем соответствующей обработки полученных экспериментальных данных (см. $\sigma T(T)$ на рис. 2) были определены параметры ионопереноса и температуры фазовых переходов фосфата натрия-скандия, которые приведены в табл. 1.

Как видно из табличных данных, низкотемпературную $\alpha$-фазу поликристалла $\mathrm{Na}_{3} \mathrm{Sc}_{2}\left(\mathrm{PO}_{4}\right)_{3}$ можно отнести к диэлектрической, т. к. она характеризуется низкими значениями проводимости $2 \cdot 10^{-5}(\Omega \cdot \mathrm{cm})^{-1}$ при $293 \mathrm{~K}$ и высокими значениями энергии активации $0.52 \mathrm{eV}$.

Полученные выше экспериментальные данные, а также результаты работы [15], в которой сообщалось о наличии доменов на поверхности кристалла $\alpha-\mathrm{Na}_{3} \mathrm{Sc}_{2}\left(\mathrm{PO}_{4}\right)_{3}$ и их исчезновении при фазовом переходе $\alpha \rightarrow \beta$, позволяют рассматривать эту фазу как сегнетоэлектрическую.

\section{3. Результаты исследования диэлектрических свойств поликристалла $\mathrm{Na}_{3} \mathrm{Sc}_{2}\left(\mathrm{PO}_{4}\right)_{3}$}

Исследование температурной зависимости диэлектрической проницаемости $\varepsilon(T)$ в диапазоне частот $5-5 \cdot 10^{5} \mathrm{~Hz}$ позволяет выявить, что низкотемпературная фаза $\alpha-\mathrm{Na}_{3} \mathrm{Sc}_{2}\left(\mathrm{PO}_{4}\right)_{3}$ является полностью дипольноупорядоченной до $320 \mathrm{~K}$, т. к. воздействие внешнего электрического поля и температуры не приводит к изменению $\varepsilon$ на зависимости $\varepsilon(T)$. При дальнейшем повышении температуры поляризационные явления в поликристалле проявлялись нечетко, особенно в области фазового перехода $T_{\alpha \rightarrow \beta}$, из-за влияния других эффектов (например, „медленных процессов“ типа ионной проводимости).

С целью исключения влияния „медленных процессов“ на поляризационные процессы в образце были проведены измерения температурной зависимости диэлектрической проницаемости $\varepsilon(T)$ на сверхвысоких частотах. В частности, на рис. 3 представлена температурная зависимость $\varepsilon(T)$ для поликристаллического образца $\mathrm{Na}_{3} \mathrm{Sc}_{2}\left(\mathrm{PO}_{4}\right)_{3}$ на частоте измерительного поля $2 \mathrm{GHz}$.

Следует отметить, что наряду с ростом диэлектрической проницаемости на зависимости $\varepsilon(T C)$ четко выделяется аномалия, соответствующая области фазового перехода $T_{\alpha \rightarrow \beta}=339 \mathrm{~K}$. Согласно закону Кюри-Вейса, для типичных сегнетоэлектриков в области фазовых 


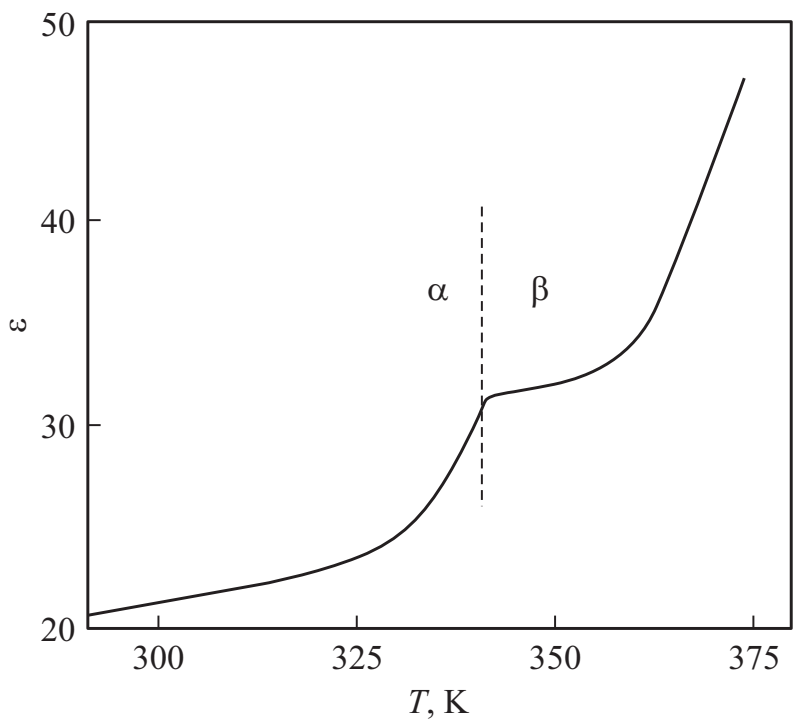

Рис. 3. Температурная зависимость диэлектрической проницаемости $\varepsilon$ для поликристаллического образца $\mathrm{Na}_{3} \mathrm{Sc}_{2}\left(\mathrm{PO}_{4}\right)_{3}$ на частоте $2 \mathrm{GHz}$

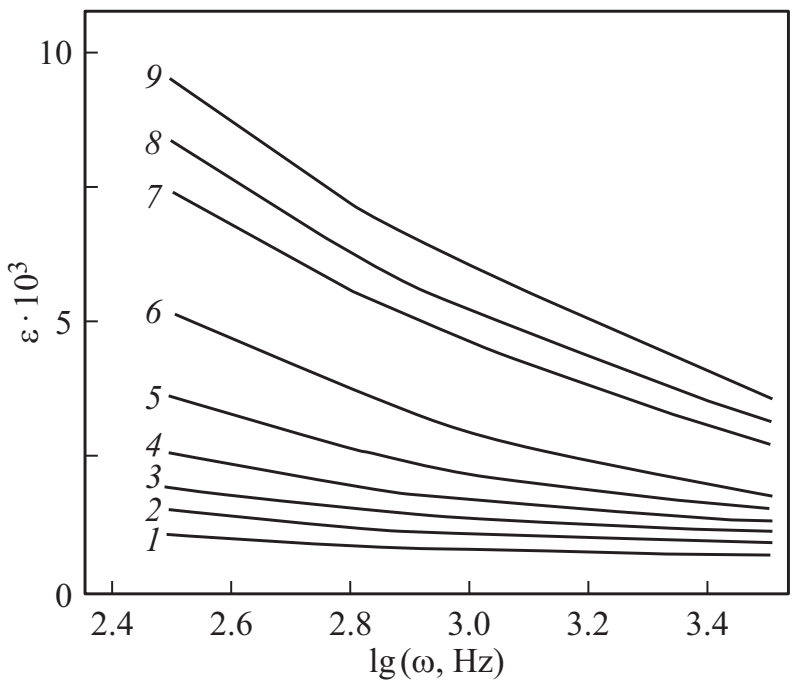

Рис. 4. Частотные зависимости диэлектрической проницаемости $\varepsilon$ для поликристаллического образца $\mathrm{Na}_{3} \mathrm{Sc}_{2}\left(\mathrm{PO}_{4}\right)_{3}$. Кривые 1-9 соответствуют измерениям, проведенным при следующих температурах: $1-293,2-313,3-323$, $4-333,5-343,6-353,7-363,8-368,9-373 \mathrm{~K}$.

переходов $\left(T_{C}\right)$ характерны пики (всплески) на температурной зависимости $\varepsilon(T)$; но установленная нами аномалия в виде „ступеньки“ на кривой $\varepsilon(T)$ позволяет классифицировать переход $T_{\alpha \rightarrow \beta}$ как несобственный сегнетоэлектрический фазовый переход, а сам образец $\alpha-\mathrm{Na}_{3} \mathrm{Sc}_{2}\left(\mathrm{PO}_{4}\right)_{3}$ - как несобственный сегнетоэлектрик [16].

Изучение частотной зависимости диэлектрической проницаемости $\varepsilon(\omega)$ низкотемпературной фазы $\alpha-\mathrm{Na}_{3} \mathrm{Sc}_{2}\left(\mathrm{PO}_{4}\right)_{3}$ показывает, что значение $\varepsilon$ практически не зависит от частоты до температур $320 \mathrm{~K}$ (см. кривые 1-4 на рис. 4). Возможно, эти результаты связаны с тем, что катионы натрия „сконденсировались“ (упорядочились в нескомпенсированные статистические натриевые диполи) на дне потенциальных ям кристаллического каркаса (на дне деформированных $B$-полостей кристаллического каркаса), из-за моноклинного искажения структуры (пр.гр. Вв), что вполне согласуется с нашими данными по проводимости и характеризует $\alpha$-фазу $\mathrm{Na}_{3} \mathrm{Sc}_{2}\left(\mathrm{PO}_{4}\right)_{3}$ как сегнетоэлектрическую (рис. 4). При изучении диэлектрических свойств $\beta-\mathrm{Na}_{3} \mathrm{Sc}_{2}\left(\mathrm{PO}_{4}\right)_{3}$ установлено, что для этой фазы поликристалла характерны релаксационные процессы поляризации, проявляющиеся плавно спадающими кривыми 5-9 на зависимости $\varepsilon(\omega)$ (рис. 4). Отметим, что с повышением температуры плавные кривые спада на зависимости $\varepsilon(\omega)$ переходят в более резко спадающие кривые (на рис. 4 видно, что угол наклона кривых 5-9 больше, чем кривых 1-4). Также релаксационные процессы можно наблюдать на частотной зависимости тангенса угла диэлектрических потерь $\operatorname{tg} \delta(\omega)$ (рис. 5).

Наиболее яркая демонстрация релаксационных максимумов проявляется на зависимости $\operatorname{tg} \delta(\omega)$ (кривые 7-9 на рис. 5).

Причем с повышением частоты наблюдается постепенная тенденция сдвига релаксационных максимумов на зависимости $\operatorname{tg} \delta(\omega)$ в область более высоких частот (от кривой 7 к кривой 9 на рис. 5). Эти данные позволяют заключить, что релаксационные процессы могут быть связаны с катионной частью кристаллического каркаса (катионами натрия), т. к. анионная часть, состоящая из полиэдров $\mathrm{ScO}_{6}, \mathrm{PO}_{4}$, составляет „жесткий“ ромбоэдрический кристаллический каркас $\left\{\left[\mathrm{Sc}_{2}\left(\mathrm{PO}_{4}\right)_{3}\right]^{3-}\right\}_{3 \infty}[2-5]$. Поэтому наблюдаемые релаксационные процессы могут

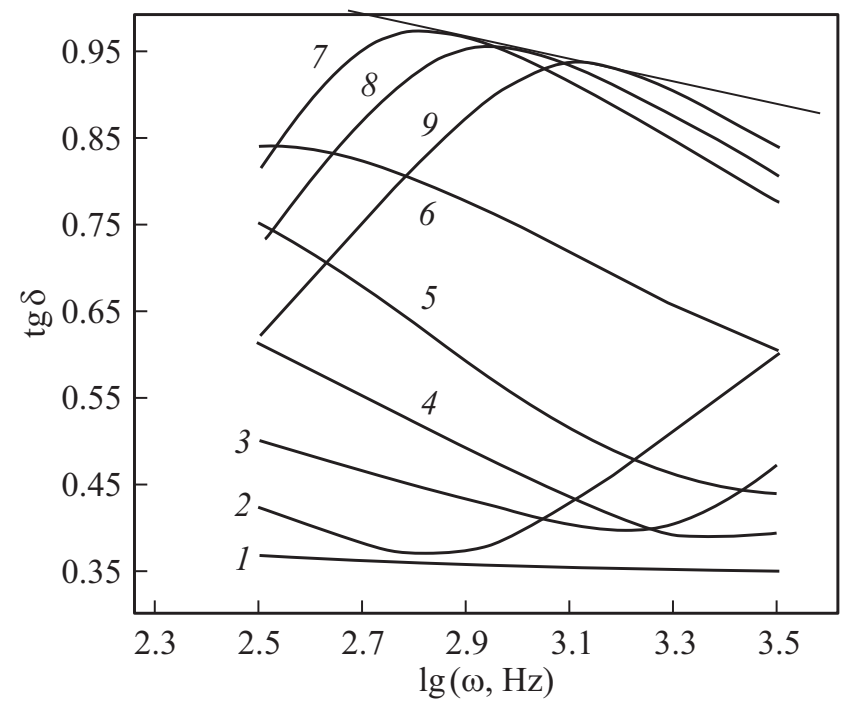

Рис. 5. Частотные зависимости тангенса угла диэлектрических потерь для поликристаллического образца $\mathrm{Na}_{3} \mathrm{Sc}_{2}\left(\mathrm{PO}_{4}\right)_{3}$. Кривые 1-9 соответствуют измерениям, проведенным при следующих температурах: $1-293,2-313,3-323$, $4-333,5-343,6-353,7-363,8-368,9-373 \mathrm{~K}$. 
быть обусловлены колебаниями слабо подвижных катионов натрия в частично деформированных $B$-полостях кристаллического каркаса $\beta-\mathrm{Na}_{3} \mathrm{Sc}_{2}\left(\mathrm{PO}_{4}\right)_{3}$ (моноклинное искажение кристаллического каркаса в $\beta$-фазе частично снято) под воздействием электрического поля и температуры выше $339 \mathrm{~K}$. Согласно результатам структурных исследований работы [3], кристаллический каркас $\beta-\mathrm{Na}_{3} \mathrm{Sc}_{2}\left(\mathrm{PO}_{4}\right)_{3}$ является ромбоэдрическим с пр.гр. $3 \bar{R} C$, но наличие дополнительных запрещенных рефлексов, зафиксированных в этой фазе, может быть признаком сверхструктурных искажений, характерных для антисегнетоэлектриков. Тогда нельзя исключить участия в релаксационном процессе и скомпенсированных натриевых диполей.

Результаты исследования проводящих и диэлектрических свойств позволяют предположить, что степень искаженности кристаллического каркаса $\beta$-фазы значительно менее выражена, чем для $\alpha-\mathrm{Na}_{3} \mathrm{Sc}_{2}\left(\mathrm{PO}_{4}\right)_{3}$, что можно связать с частичным снятием искаженности кристаллического каркаса и появлением подвижных зарядов. С этими изменениями можно связать наблюдаемую на рис. 5 релаксационную поляризацию ионов натрия в $\beta-\mathrm{Na}_{3} \mathrm{Sc}_{2}\left(\mathrm{PO}_{4}\right)_{3}$.

При последующем фазовом переходе $\beta \rightarrow \gamma$ релаксационные процессы резко ускоряются и сдвигаются в область более высоких частот (в силу ограниченности приборных возможностей экспериментально не представлено). Появление быстрых релаксационных процессов может быть связано с резким повышением концентрации подвижных катионов натрия в кристаллическом каркасе образца, из-за полного дипольного разупорядочения.

Вероятно, наблюдаемые пики на частотной зависимости $\operatorname{tg} \delta(\omega)$ для $\beta-\mathrm{Na}_{3} \mathrm{Sc}_{2}\left(\mathrm{PO}_{4}\right)_{3}$ связаны с релаксационными процессами поляризации дебаевского типа (показано кривой 1 на рис. 6), т. к. экспериментальная характеристика образца, показанная кривой 1 , близка к теоретической дебаевской кривой 2 на рис. 6, построение которой осуществлялось согласно формуле Дебая (2) [17]:

$$
\operatorname{tg} \delta=\frac{\sigma}{\omega}+\frac{\left(\varepsilon_{0}-\varepsilon_{\infty}\right)}{\varepsilon_{0}+\varepsilon_{\infty} \omega^{2} \tau^{2}},
$$

где $\tau$ - время релаксации диполя в диэлектрике под воздействием внешнего поля; $\varepsilon_{0}$ и $\varepsilon-$ статическая и оптическая диэлектрические проницаемости соответственно; $\sigma$ - ионная проводимость.

Однако имеющиеся различия между экспериментальной (кривая 1) и расчетной (кривая 2) зависимостями на частотной характеристике $\operatorname{tg} \delta(\omega)$ (рис. 6) могут быть связаны с тем, что в релаксационном процессе в $\beta-\mathrm{Na}_{3} \mathrm{Sc}_{2}\left(\mathrm{PO}_{4}\right)_{3}$ участвуют как свободные катионы натрия (преимущественно), так и скомпенсированные натриевые диполи (не исключатся их слабое участие).

В целом параметры процесса релаксационной поляризации для $\beta$-фазы этого соединения были определены путем анализа частотной зависимости тангенса угла диэлектрических потерь (см. рис. 5 и 6). Дополнительные

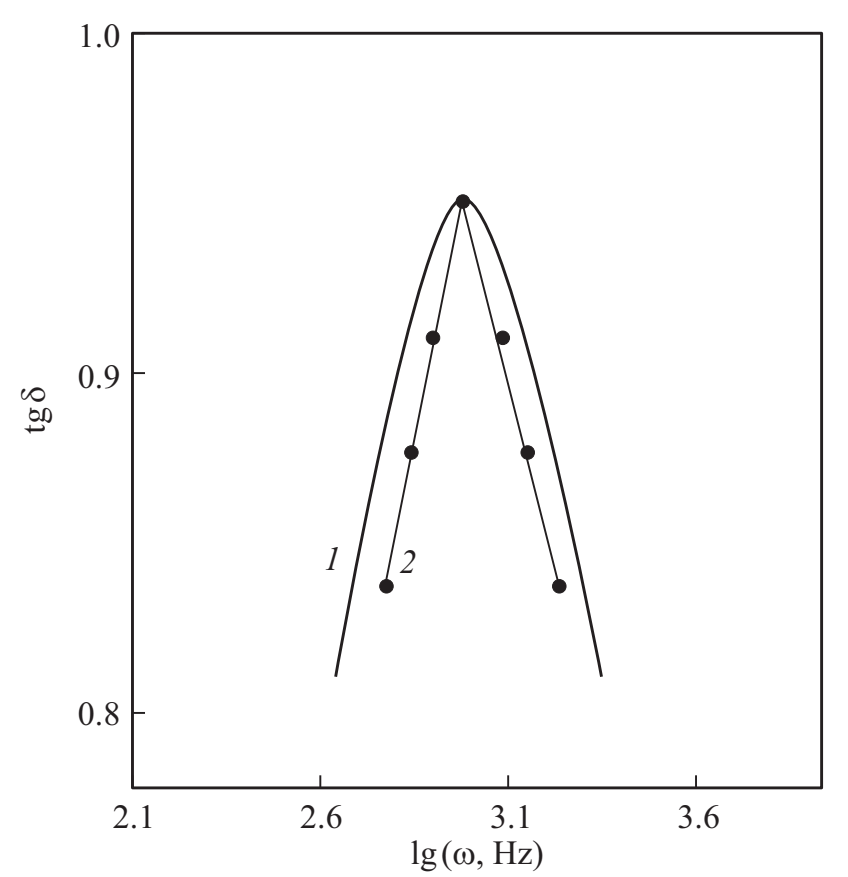

Рис. 6. Частотные зависимости тангенса угла диэлектрических потерь $\delta$ для поликристаллического образца $\mathrm{Na}_{3} \mathrm{Sc}_{2}\left(\mathrm{PO}_{4}\right)_{3}$ при температуре $T=368 \mathrm{~K} .1-$ экспериментальная кривая, 2 - теоретическая кривая.

параметры тепловой релаксационной поляризации для $\beta-\mathrm{Na}_{3} \mathrm{Sc}_{2}\left(\mathrm{PO}_{4}\right)_{3}$ определены путем построения зависимости $\omega_{\max }(T)$. Были определены энергия активации $\Delta E$ и среднее время релаксации $\tau$ при нулевой температуре для этой фазы. Тогда время релаксации $\tau$ может быть определено согласно [17] в виде:

$$
\tau=\frac{1}{2} v \exp (\Delta E / k T)
$$

где $v$ - частота собственных колебаний диполей; $\exp (\Delta E / k T)$ отражает вероятность преодоления дипольной частицей потенциального барьера высотой $\Delta E$, разделяющего диполи в их устойчивом состоянии; $k-$ постоянная Больцмана. Параметры релаксационной поляризации для $\mathrm{Na}_{3} \mathrm{Sc}_{2}\left(\mathrm{PO}_{4}\right)_{3}$ приведены в таблице 2 .

Учитывая установленные структурные данные для $\alpha-\mathrm{Na}_{3} \mathrm{Sc}_{2}\left(\mathrm{PO}_{4}\right)_{3}$ и результаты работ [1-5], а также ее частотные характеристики $\varepsilon(\omega)$ и $\operatorname{tg} \delta(\omega)$, представленные на рис. 4 и 5, можно заключить, что дипольное упорядочение катионов натрия в $\alpha-\mathrm{Na}_{3} \mathrm{Sc}_{2}\left(\mathrm{PO}_{4}\right)_{3}$ представляет собой систему связанных, малоподвижных диполей в $B$-полости кристаллического каркаса. Основываясь на специфике заселенности катионов натрия в $A$ - и $B$-полостях кристаллического каркаса $\mathrm{Na}_{3} \mathrm{Sc}_{2}\left(\mathrm{PO}_{4}\right)_{3}$ [2-5], можно говорить об образовании нескомпенсированных статистических натриевых диполей в $B$-полостях кристаллического каркаса $\left\{\left[\mathrm{Sc}_{2}\left(\mathrm{PO}_{4}\right)_{3}\right]^{P-}\right\}_{3 \infty}$.

Согласно [5], в низкотемпературной фазе $\alpha-\mathrm{Na}_{3} \mathrm{Sc}_{2}\left(\mathrm{PO}_{4}\right)_{3}$, атомами натрия заселены только 
Таблица 2. Параметры релаксационной поляризации для $\mathrm{Na}_{3} \mathrm{Sc}_{2}\left(\mathrm{PO}_{4}\right)_{3}$

\begin{tabular}{l|c|c|c}
\hline \multicolumn{1}{c|}{ Параметры $\mathrm{Na}_{3} \mathrm{Sc}_{2}\left(\mathrm{PO}_{4}\right)_{3}$} & \multicolumn{3}{c}{ Фазы } \\
\cline { 2 - 4 } & \multicolumn{1}{c}{$\beta$} & $3 \bar{R} C$ \\
\hline Симметрия & 86 & $9 \cdot 10^{3}$ при $440 \mathrm{~K}$ \\
\hline Диэлектрические проницаемости $\varepsilon$ на частоте $1259 \mathrm{~Hz}$ & $8 \cdot 10^{2}$ при $293 \mathrm{~K}$ & $5 \cdot 10^{3}$ при $368 \mathrm{~K}$ & \\
\hline $\begin{array}{l}\text { Максимум тангенса угла диэлектрических потерь } \operatorname{tg} \delta_{\max } \\
\text { на частоте } 1259 \mathrm{~Hz}\end{array}$ & & 0.95 при $368 \mathrm{~K}$ & \\
\hline Энергия активации процесса релаксации $\Delta E, \mathrm{eV}$ & - & 0.33 & \\
\hline Время релаксации $\tau, \mathrm{s}$ & - & $1.6 \cdot 10^{-5}$ &
\end{tabular}

$B$-полости, причем в одной трети $B$-полости атомы натрия располагаются почти в центрах, а в оставшихся двух третьих полости атомы натрия сдвинуты из центров на $0.39,1.64,0.75$ и $2.11 \AA$ в сторону одной из $A$-полостей, что приводит к образованию двух позиций $B 1-B 2$ или $C 1-C 2$ статистически заселенных катионами натрия с кратностями, различающимися в 1.7 раза. Не исключено, что именно такой порядок заполнения катионов натрия в анионном кристаллическом каркасе приводит к возникновению спонтанно-поляризованного состояния, с образованием в кристалле систем натриевых диполей, упорядоченных в соответствии с пр.гр. Вв.

В случае $\alpha-\mathrm{Na}_{3} \mathrm{Sc}_{2}\left(\mathrm{PO}_{4}\right)_{3}$ [2,3,5], статистические натриевые диполи образовывались за счет неравномерного заселения катионов натрия в $B$-полостях кристаллического каркаса, вызванного расщеплением натриевых позиций $\mathrm{Na}_{2}$ на две неравноценные части. Вероятно, появление таких натриевых диполей и приводит к смещению центра суммарных положительных зарядов (катионов компенсаторов натрия) относительно центра $B$-полости кристаллического каркаса. С другой стороны, в центре $B$-полости должен фокусироваться центр отрицательных зарядов, создающихся анионным кристаллическом каркасом $\left\{\left[\mathrm{Sc}_{2}\left(\mathrm{PO}_{4}\right)_{3}\right]^{P-}\right\}_{3 \infty}$.

В результате можно говорить о „разделении“ центров положительных и отрицательных зарядов вокруг центра $B$-полости кристаллического каркаса, что эквивалентно образованию в кристалле виртуальных „натриевых диполей“ (иначе - статистических натриевых диполей).

Эти представления согласуются с экспериментальным результатами (нецентросимметричности кристалла при $T=293 \mathrm{~K}$, проводящих и диэлектрических свойств), установленными для поликристалла $\alpha-\mathrm{Na}_{3} \mathrm{Sc}_{2}\left(\mathrm{PO}_{4}\right)_{3}$.

\section{4. О моделях потенциального барьера и релаксатора для $\alpha-, \beta-\mathrm{Na}_{3} \mathrm{Sc}_{2}\left(\mathrm{PO}_{4}\right)_{3}$}

Системы, для которых релаксация на микроскопическом уровне может быть описана уравнением (4), применима модель глубокой потенциальной ямы с двумя положениями равновесия, или релаксатор Флериха

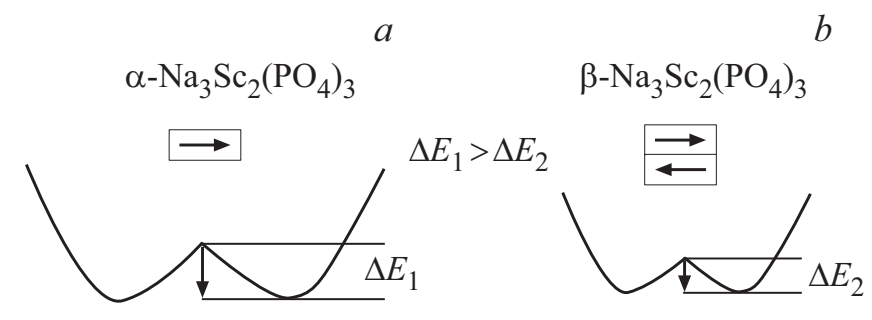

Рис. 7. Схематическое изображение потенциального барьера и характера дипольного упорядочения для $\alpha$ - и $\beta$ - $\mathrm{Na}_{3} \mathrm{Sc}_{2}\left(\mathrm{PO}_{4}\right)_{3}$ : $a)$ изображение потенциальной ямы с двумя положениями равновесия и изображение нескомпенсированного натриевого диполя для $\alpha$-фазы; $b$ ) изображение потенциальной ямы и скомпенсированного натриевого диполя для $\beta$-фазы.

(рис. 7,a). Основываясь на этой модели и модели, предложенной для диэлектрической фазы $\alpha-\mathrm{Na}_{3} \mathrm{Sc}_{2}\left(\mathrm{PO}_{4}\right)_{3}$ в $[2,6]$, а также данных по исследованию проводящих диэлектрических свойств и структурных параметров $\alpha$ - и $\beta-\mathrm{Na}_{3} \mathrm{Sc}_{2}\left(\mathrm{PO}_{4}\right)_{3}$, может быть предложена модель двухминимумного потенциального рельефа вдоль канала проводимости (рис. 7). Следует отметить, что глубина потенциального рельефа (энергетическая характеристика) в модели для $\alpha-\mathrm{Na}_{3} \mathrm{Sc}_{2}\left(\mathrm{PO}_{4}\right)_{3}$ больше (см. рис. 7, $a$ ), чем в $\beta-\mathrm{Na}_{3} \mathrm{Sc}_{2}\left(\mathrm{PO}_{4}\right)_{3}$ (см. рис. $\left.7, b\right)$, из-за различия в степени структурных искажений кристаллического каркаса.

В случае $\alpha$-фазы релаксаторами при релаксационной поляризации могут быть нескомпенсированные статистические натриевые диполи (см. рис. 7,a), а в случае $\beta-\mathrm{Na}_{3} \mathrm{Sc}_{2}\left(\mathrm{PO}_{4}\right)_{3}$, релаксаторами могут являться как разупорядоченные ионы натрия (см. рис. 7,b), так и скомпенсированные статистические натриевые диполи (с учетом, обнаруженных в этой фазе сверхструктурных искажений). На рис. 7, $b$ схематически изображен скомпенсированный натриевый диполь, образующийся из двух противоположно направленных нескомпенсированных статистических натриевых диполей, вследствие удвоения объема элементарной ячейки кристалла.

Вероятно, процесс релаксационной поляризации, вызванный перемещением ионов натрия в полостях 
ромбоэдрического кристаллического каркаса $\left\{\left[\mathrm{Sc}_{2}\left(\mathrm{PO}_{4}\right)_{3}\right]^{3}\right\}_{3 \infty}$, эквивалентен перебросу как катионов натрия, так и скомпенсированных статистических натриевых диполей через потенциальный барьер $E=0.33 \mathrm{eV}$ за среднее время $\tau=1.6 \cdot 10^{-5} \mathrm{~s}$.

\section{5. О поляризационных процессах в $\beta$ - и $\gamma$-фазах $\mathrm{Na}_{3} \mathrm{Sc}_{2}\left(\mathrm{PO}_{4}\right)_{3}$}

Переход в $\beta$-фазу сопровождается частичным разрушением статистических натриевых диполей в $\beta$ - $\mathrm{Na}_{3} \mathrm{Sc}_{2}\left(\mathrm{PO}_{4}\right)_{3}$, о чем свидетельствует высокая ионная проводимость, скачок на температурной зависимости проводимости (см. рис. 1), а также установленные диэлектрические и структурные параметры.

Отметим, что $\beta$ - $\mathrm{Na}_{3} \mathrm{Sc}_{2}\left(\mathrm{PO}_{4}\right)_{3}$ имеет квазиромбоэдрическое строение $[2,5]$. Все результаты по исследованию структуры и тепловых релаксационных процессов вполне согласуются с тем, что релаксаторами могут быть как „свободные“ ионы натрия, так и скомпенсированные натриевые диполи.

Для $\gamma-\mathrm{Na}_{3} \mathrm{Sc}_{2}\left(\mathrm{PO}_{4}\right)_{3}$, максимумы тангенса угла диэлектрических потерь сдвинуты в область более высоких частот и выходят за пределы частотного диапазона наших исследований. Очевидно, что процесс релаксационной поляризации для $\gamma$ - $\mathrm{Na}_{3} \mathrm{Sc}_{2}\left(\mathrm{PO}_{4}\right)_{3}$ сдвигается в область более высоких частот. Вполне логично появление таких поляризационных процессов связать с быстрыми колебаниями разупорядоченных ионов натрия в кристаллическом каркасе $\gamma-\mathrm{Na}_{3} \mathrm{Sc}_{2}\left(\mathrm{PO}_{4}\right)_{3}$ под действием внешнего электрического поля.

Усиление быстрых поляризационных процессов при переходе в ромбоэдрическую фазу $\gamma-\mathrm{Na}_{3} \mathrm{Sc}_{2}\left(\mathrm{PO}_{4}\right)_{3}$ с пр. гр. $3 \bar{R} C$ может быть связано с максимальным повышением симметрии ромбоэдрического кристаллического каркаса и полным разупорядочением скомпенсированных статистических натриевых диполей. Высокая подвижность и равномерное распределение ионов натрия по $A$ - и $B$-полостям ромбоэдрического кристаллического каркаса создают все условия для протекания быстрых поляризационных процессов под действием высокочастотного электрического поля.

\section{6. О модели дипольного упорядочения в $\alpha$-фазе и ионной проводимости в $\beta$ - и $\gamma$-фазах $\mathrm{Na}_{3} \mathrm{Sc}_{2}\left(\mathrm{PO}_{4}\right)_{3}$}

В работах [2-10] показано, что процессы структурного разупорядочения при фазовых переходах $\alpha \rightarrow \beta, \beta \rightarrow \gamma$ в $\mathrm{Na}_{3} \mathrm{Sc}_{2}\left(\mathrm{PO}_{4}\right)_{3}$ приводят к качественным изменениям их проводящих свойств.

В $[2,5,6,8]$ показано, что основой кристаллической структуры $\mathrm{Na}_{3} \mathrm{Sc}_{2}\left(\mathrm{PO}_{4}\right)_{3}$ является ажурный кристаллический каркас $\left\{\left[\mathrm{Sc}_{2}\left(\mathrm{PO}_{4}\right)_{3}\right]^{3-}\right\}_{3}$, характеризующийся наличием трехмерных каналов. Эти каналы образуются благодаря сочленению полостей $A$ - и $B$-типов через гофрированные гексагональные кольца, причем $A$-полости

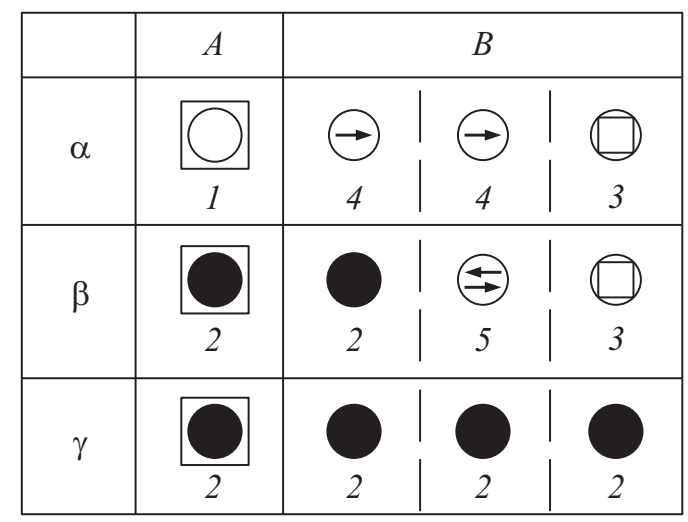

Рис. 8. Кристаллохимическая модель, поясняющая условное распределение атомов натрия по позициям в $A$ - и $B$-полостях кристаллического каркаса $\alpha$-, $\beta$-, $\gamma$ - $\mathrm{Na}_{3} \mathrm{Sc}_{2}\left(\mathrm{PO}_{4}\right)_{3}: 1$ - вакантная полость, 2 - статистическое заселение позиций катионами натрия, 3 - заселение катионов натрия с упорядочением, 4 - нескомпенсированный статистический натриевый диполь, 5 - скомпенсированный статистический натриевый диполь.

меньше по размеру, чем $B$-полости. Для $\alpha-\mathrm{Na}_{3} \mathrm{Sc}_{2}\left(\mathrm{PO}_{4}\right)_{3}$ катионы натрия упорядочены в статистические натриевые диполи в $B$-полостях, вследствие моноклинного искажения кристаллического каркаса. При переходе в высокотемпературные $\beta$-, $\gamma$-фазы атомы натрия могут быть статистически распределены как в $A$-, так и в $B$-полостях, что создает благоприятные условия для проявления высокой ионной проводимости.

Установленные кардинальные изменения электрофизических свойств при фазовых превращениях и соответствующие структурные изменения кристаллического каркаса в $\alpha-\mathrm{Na}_{3} \mathrm{Sc}_{2}\left(\mathrm{PO}_{4}\right)_{3}$ в пределах небольшого температурного интервала $(T=293-573 \mathrm{~K})$ позволяют говорить об исключительной „эластичности“ кристаллического каркаса данного типа.

Для наглядной демонстрации изменений электрофизических свойств $\mathrm{Na}_{3} \mathrm{Sc}_{2}\left(\mathrm{PO}_{4}\right)_{3}$ в зависимости от фазовых состояний $(\alpha, \beta, \gamma)$ предложена уточненная кристаллохимическая модель (за основу взята модель, описанная в работе [5]), изображенная на рис. 8.

Распределение атомов натрия по $A$ - и $B$-полостям кристаллического каркаса для $\alpha$-, $\beta$-, $\gamma$-фаз $\mathrm{Na}_{3} \mathrm{Sc}_{2}\left(\mathrm{PO}_{4}\right)_{3}$ может быть представлено схематически так, как изображено на рис. 8. При этом $A$-полости кристалла могут быть вакантными или статистически заняты атомами натрия, а $B$-полости заняты статистически с упорядочением, либо в виде статистических натриевых диполей, либо заселены равномерно катионами натрия.

Факт наличия вакантных $A$-полостей изображен на рис. 8 светлым кругом, вписанным в квадрат, и пронумерован позицией 1 , а присутствие упорядоченных состояний и нескомпенсированных статистических натриевых диполей в кристаллических каркасах $\alpha-\mathrm{Na}_{3} \mathrm{Sc}_{2}\left(\mathrm{PO}_{4}\right)_{3}$ пронумерованы позициями 3 и 4 соответственно (см. рис. 8). Статистическое заселение $A$ - и $B$-полостей 
кристаллического каркаса в $\alpha-, \beta-, \gamma-\mathrm{Na}_{3} \mathrm{Sc}_{2}\left(\mathrm{PO}_{4}\right)_{3}$ пронумерованы позицией 2 (все $A$-полости изображены в виде окружностей, вписанных в квадрат).

Однако следует обратить внимание, что поликристалл $\alpha-\mathrm{Na}_{3} \mathrm{Sc}_{2}\left(\mathrm{PO}_{4}\right)_{3}$ является несобственным сегнетоэлектриком (показано верхней строкой таблицы). Согласно данной модели, для $\alpha-\mathrm{Na}_{3} \mathrm{Sc}_{2}\left(\mathrm{PO}_{4}\right)_{3}$ характерны как упорядоченные (позиции 3 на рис. 8), так и дипольноупорядоченные состояния в $B$-полости кристаллического каркаса (позиции 4 и 5 на рис. 8). При этом $A$-полости полностью вакантны.

Для $\beta-\mathrm{Na}_{3} \mathrm{Sc}_{2}\left(\mathrm{PO}_{4}\right)_{3}$ вполне логично представить, что части $B$-полостей будут заселены свободными катионами натрия в виде позиций 2 , а также скомпенсированными статистическими натриевыми диполями, которые пронумерованы в виде позиции 5 (см. рис. 8).

При фазовых переходах $\alpha \rightarrow \beta, \beta \rightarrow \gamma$ в поликристалле $\mathrm{Na}_{3} \mathrm{Sc}_{2}\left(\mathrm{PO}_{4}\right)_{3}$ происходят акты последовательного повышения симметрии поликристалла, приводящие к разрушению как упорядоченных, так и дипольноупорядоченных состояний, и к более равномерному статистическому распределению катионов натрия по $A$ и $B$-полостям кристаллического каркаса, как показано для $\gamma-\mathrm{Na}_{3} \mathrm{Sc}_{2}\left(\mathrm{PO}_{4}\right)_{3}$ на рис. 8 .

\section{4. Выводы}

На основе представленных экспериментальных результатов можно сделать следующие выводы:

1) Доказано, что структура поликристалла $\alpha-\mathrm{Na}_{3} \mathrm{Sc}_{2}\left(\mathrm{PO}_{4}\right)_{3}$ обладает упорядочением сегнетоэлектрического типа, а фазовый переход $\alpha \rightarrow \beta$ может быть отнесен к несобственному сегнетоэлектрическому переходу. При фазовых переходах $\alpha \rightarrow \beta$, $\beta \rightarrow \gamma$ в поликристалле $\mathrm{Na}_{3} \mathrm{Sc}_{2}\left(\mathrm{PO}_{4}\right)_{3}$ происходят акты последовательного повышения симметрии кристалла и ионной проводимости. Установлено, что в диэлектрической фазе $\beta$ - $\mathrm{Na}_{3} \mathrm{Sc}_{2}\left(\mathrm{PO}_{4}\right)_{3}$ тепловая релаксационная поляризация носит дебаевский характер, причем релаксаторами являются как разупорядоченные катионы натрия, так и скомпенсированные натриевые диполи. Поликристалл $\beta-\mathrm{Na}_{3} \mathrm{Sc}_{2}\left(\mathrm{PO}_{4}\right)_{3}$ можно отнести к ионному проводнику, хотя в $B$-полостях кристаллического каркаса не исключено наличие скомпенсированных статистических натриевых диполей.

2) Установлено, что поликристалл $\gamma-\mathrm{Na}_{3} \mathrm{Sc}_{2}\left(\mathrm{PO}_{4}\right)_{3}$ является суперионным проводником, вследствие высоких ионно-проводящих свойств и полной симметризации кристаллического каркаса. Для $\beta$-, $\gamma$-фаз характерны быстрые релаксационные процессы поляризации, обусловленные наличием подвижных катионов натрия в $A$ и $B$-полостях кристаллического каркаса.

3) Предложена кристаллохимическая модель, поясняющая явления дипольного упорядочения и ионной проводимости в $\mathrm{Na}_{3} \mathrm{Sc}_{2}\left(\mathrm{PO}_{4}\right)_{3}$.
4) Наличие в литературных источниках множества противоречивых сведений, касающихся структурных и проводящих параметров, а также температур фазовых превращений в двойном фосфате натрия-скандия можно связать с ажурностью трехмерного каркаса $\left\{\left[\mathrm{Sc}_{2}\left(\mathrm{PO}_{4}\right)_{3}\right]^{3}\right\}_{3 \infty}$, который допускает широкий спектр форм кристаллизации образца в зависимости от термодинамического режима и условий синтеза.

\section{Конфликт интересов}

Авторы заявляют, что у них нет конфликта интересов.

\section{Список литературы}

[1] А.С. Ногай, С.Ю. Стефанович, А.А. Буш, Д.Е. Ускенбаев, А.А. Ногай. ФТТ 60, 25 (2018).

[2] В.А. Ефремов, В.Б. Калинин. Кристаллография 20, 703 (1978).

[3] Б.И. Лазоряк, В.Б. Калинин, С.Ю. Стефанович, В.А. Ефремов. ДАН СССР 250, 861 (1980).

[4] С.Ю. Стефанович, В.Б. Калинин. ФТТ 23, 3509 (1981).

[5] В.Б. Калинин, Б.И. Лазоряк, С.Ю. Стефанович. Кристаллография 28, 264 (1983).

6] B. Susman, C. Delbecq, D.T.O. Brun, E. Princo. Solid State Ionics 9-10, 839 (1983).

[7] Л.О. Атовмян, Н.Г. Букун, В.И. Коваленко, А.И. Коростелева, В.В. Ткачев, Е.А. Укше. Электрохимия 19, 933 (1983).

[8] В.Б. Калинин, С.Ю. Стефанович, А.С. Ногай. Неорган. материалы 22, 107 (1986).

[9] А.С. Ногай, Yung Huh, К.Н. Югай. ФТТ 47, 1042 (2005).

[10] L. Vijayan, R. Cheruku, G. Govindaraj, S. Rajagopan. Mater. Chem. Phys. 130, 862 (2011).

[11] Н.И. Сорокин. ФТТ 56, 652 (2014).

[12] D. Rettenwander, G.J. Redhammer, M. Guin, A. Benisek, H. Krüger, O. Guillon, M. Wilkening, F. Tietz, J. Fleig. Chem. Mater. 30, 1776 (2018)

[13] L.B. Ellis, F. Linda. Current Opinion Solid State Mater. Sci. 16, 168 (2012).

[14] В.Б. Ткачев, В.И. Пономарев, Л.О. Атовмян. ЖСХ 25, 128 (1984).

[15] С.А. Оконенко, С.Ю. Стефанович, В.Б. Калинин, Ю.Н. Веневцев. ФТТ 20, 2846 (1978).

[16] Б.А. Струков, А.П. Леванюк. Физические основы сегнетоэлектрических явлений в кристаллах. Наука, М. (1983), $240 \mathrm{c}$.

[17] Ю.М. Поплавко. Физика диэлектриков. Высш. шк. Киев (1980), $398 \mathrm{c}$.

Редактор Е.В. Толстякова 